\title{
Ressenyes
}

\section{Violeta DÍAZ-CoRRALEJO}

\section{Los gestos en la literatura medieval} Madrid: Editorial Gredos, 2004

Ricostruire, all'interno della civiltà letteraria medievale, i parametri interpretativi di un linguaggio corporale, quello dei gesti e dei movimenti - che rispondono in ogni cultura a precise e determinate esigenze di trasmissione di un messaggio - costituisce il nucleo dello studio di Violeta Díaz-Corralejo. Frammenti di una comunicazione pre-verbale, i gesti sono interpretati dall'autrice come la sede di una trasmissione di significati alternativa a quella linguistica, come valore aggiunto ad essa, che comporta la produzione di un messaggio spesso preciso e interpretabile.

Indispensabile per una più ampia comprensione dei testi letterari e soprattutto medievali, la studiosa sperimenta una metodologia di ricerca che delimita entro un campo di indagine relativo alla medievalistica ma applicabile «a cualquier otra época de la literatura». In particolare, il corpus esaminato da Violeta DíazCorralejo è costituito dall'Inferno di Dante, la cantica più ricca di gestualità all'interno della Commedia. L'analisi sistematica del linguaggio corporale infernale è finalizzato ad una sua interpretazione allegorica sulla base epistemologica fornita, da un lato, dalla riflessione sul significato dei gesti tardo-antica e medievale e dall'altro dai risultati degli studi sui programmi iconografici ed ideologici forniti dalle discipline storico-artistiche e antropologiche. Il repertorio dei significati gestuali convenzionali, così come la sintassi iconografica delle miniature, costituisce per l'autrice una «civilización de los gestos», un repertorio di messaggi audiovisuali interpretabili innanzitutto mediante lo studio dei testi teorici e filosofici che lo codificarono e interpretarono da Platone alla scolastica. Nel XIII secolo, per la medievalista, si pongono le basi teoriche per una nuova interpretazione delle movenze corporee, dovuta allo studio dell'etica aristotelica, che diventa la base morale della scolastica ed al nuovo metodo sperimentato dalla scienza medica sulla scorta della fisica aristotelica.

Manifestazione agostiniana degli stati d'animo, i gesti visualizzano la dialettica corpo-anima, nell'individuo e quella più generica tra interno-esterno nella società. Il controllo del gesto, che per Alcuino equivale ad una forma di controllo sull'anima, è inoltre, nella seconda metà del XI secolo, un segno di distinzione sociale nonché una delle sedi della costruzione del rapporto con l'altro e quindi della relazione sociale tra individui; «el carácter 
ritualizado de la sociedad medieval» fa sì che il gesto definisca la persona e la identifichi con un gruppo sociale. Mostrando l'aspetto esterno dei movimenti interni, il gesto è per Ugo di San Vittore un signum, indicium e modum, che manifesta l'armonia interiore, ma è anche, per S. Tommaso, il luogo di una possibile simulatio. Díaz-Corralejo auspica e promuove un'applicazione dei principi della decodificazione relativi ai programmi iconografici di tipo politico e religioso ai testi letterari, dal momento che l'espressione di un messaggio attraverso l'uso di una determinata parte del corpo riveste una funzione spesso importante nell'elaborazione di un personaggio e nella descrizione della cultura cui partecipa. L'interpretazione gestuale disvela un mondo di simboli e allegorie interpretati dall'autrice nella prospettiva ermeneutica cui i risultati di anni di ricerche dei dantisti dell'Asociación Complutense de Dantología hanno da tempo abituato i lettori della rivista Tenzone. La studiosa fa parte, infatti, del gruppo che da qualche anno edita la prima rivista di filologia ed esegesi dantesca in Spagna - e del Seminario di studi danteschi - promosso dal prof. Carlos Lopez-Cortezo - la cui più che decennale attività si riflette nella prospettiva metodologica che informa molti dei contributi presenti nella rivista. Gli italianisti dell'Asociación Complutense de Dantología hanno, infatti, lavorato per molti anni all'analisi sistematica delle similitudini nella Commedia ed alla loro interpretazione allegorica Violeta DíazCorralejo dedica un ampio spazio all'interno del testo. Sia Carlos Lopez-Cortezo, fondatore dell'Asociación de Dantología e della rivista, che gli altri dantisti che di essa fanno parte, come Juan Varela e Rosario Scrimieri, hanno dedicato allo studio delle similitudini dantesche importanti monografie che costituiscono oggi un punto di riferimento centrale per il dantismo spagnolo. Se quindi le scelte metodologiche e quelle riguardanti il cor- pus analizzato affondano le proprie radici nelle attività esegetiche appena ricordate anche il «marco general de referencia» per interpretare il significato dei gesti nella narrazione della Commedia va individuato nella stessa prospettiva: la Commedia come visione e l'Inferno come regolato da una struttura morale e da una propria dinamica del peccato. Aristotele, Riccardo di San Vittore e Tommaso d'Aquino sono le auctoritates invocate per l'interpretazione allogorica generale dell'opera. Come le similitudini, i gesti costruiscono un canale di comunicazione alternativo e complementare che aggiunge contenuti a quelli già conosciuti ed arricchisce la comprensione del testo di più profondi significati. La dantista analizza, per ovvie ragioni di spazio, solo pochi canti, scelti come significativi delle tre zone dell'Inferno che corrispondono alle tre attitudini peccaminose di cui sono vittime gli abitanti dell'oltretomba. Il VI canto sarà, quindi, scelto per illustrare la zona dell'incontinenza, il IX per descrivere il passaggio dalla prima zona a quella della violenza e il gruppo dei canti costituiti dal XXI-XXII-XXIII, in piene Malebolge, esemplificano la funzione gestuale nel regno della frode. Sono tutti canti noti o per l'alto contenuto morale che incarnano o per la funzione che esplicitano all'interno dello sviluppo diegetico della Commedia. Il VI è il canto politico per eccellenza dell'Inferno come lo sono, notoriamente i sesti canti di tutta l'opera - il IX si contraddistingue per la ricchezza della presenza di figure mitologiche di memoria classica e cristiana che evidenziano il forte contenuto simbolico della discesa verso il regno della matta bestialitate ed infine il gruppo di canti delle malebolge, uno degli episodi della Commedia col più alto numero sia di similitudini che di gesti, da' la possibilità all'autrice di portare avanti un'accurata analisi della gesticulatio diabolica. Dopo un'attenta lectura dei canti indicati come rappresentativi per la ricogni- 
zione allegorico-gestuale, in cui le espressioni corporee sono analizzate all'interno del contesto narrativo di riferimento, si passa ad un'analisi dettagliata dei gesti infernali ora decontestualizzati rispetto alla cornice narrativa, isolati e valutati in sé. L'analisi sistematica da' vita ad un'ampia e dettagliata casistica in cui la fenomenologia delle figure e dei movimenti fisici viene descritta mediante la distinzione tra le parti del corpo che originano un determinato movimento. Testa, volto, occhi, braccia, mani, gambe e piedi sono gli attori principali di un repertorio preverbale analizzato minuziosamente e posto in essere dai protagonisti del viaggio oltremondano: Dante, Virgilio, i condannati, i diavoli, i guardiani e le altre inquietanti presenze del regno dei morti. In totale i gesti dell'Inferno sono 319, la maggior parte di essi riguarda i peccatori. Ne risulta un'evidente relazione tra gestualità e peccato, soprattutto considerando che l'organo che in questo caso è il più presente nella produzione dei gesti sono le mani, che il peccato, appunto, commisero. Segue Dante, come maggior produttore di gestualità, ma l'organo più sollecitato è, nel caso del protagonista, quello della vista. Nel cammino iniziatico verso la visione finale della verità lo sguardo, allegoria della conoscenza, è il vero protagonista della mimica corporale dantesca, mentre la zona che si distingue per il tasso più alto di espressioni nonverbali è quella delle malebolge, il regno della frode. Lo studio dei gesti, conclude Violeta Díaz-Corralejo, può quindi fornire una visione distinta o più completa dei canti infernali mediante la decodificazione di un canale di trasmissione di senso alternativo a quello linguistico e che, interpretato in una prospettiva esegetico-allegorica, può, come lo studio delle similitudini, fornire importanti informazioni per una più completa, $\mathrm{o} a$ volte diversa e nuova, lectura del testo dantesco.

\section{Chiara Cappuccio}

\section{Francesco FURLAN}

\section{Studia albertiana. Lectures et lecteurs de L. B. Alberti}

Torino-Paris: Nino Aragno Editore - J. Vrin, 2003.

Alcuni anni di lavoro attorno alla figura e alla cultura di Alberti sono raccolti da F. in questo ampio volume, nel segno della definizione di un progetto globale di scelta dalla parte umanistica intesa a un sapere concreto, a una scienza non superflua né superficiale oltreché da subito schierata - e sono le pagine prime dell'opera in argomento - contro i valori esteriori (contro l'alchimia, ad es.) e pure in nome di un ideale di utilitas che è tanto dinamico quanto ancorato a fondamenti di certezza epistemologica e tout court scientifica. Lintuizione del dialogo come genere-contenitore meglio adatto alla discussione su questi ordini di faccende viene dunque considerata, e giustamen- te, da F. come luogo qualificante un intero, lucido versante del programma umanistico di Alberti, dove la storia tecnica dello strumento dialogico - un ibrido anche per gli antichi, Luciano in primis docente - viene fatta ascendere alle stesse difficoltà discretive denunciate da Platone in merito ai rapporti del genere col criterio fondamentale della mimesi: allo specchio dunque di una riflessione lunga diversi secoli che col principio medesimo di credibilità storica aveva dovuto fare $\mathrm{i}$ conti $a b$ ovo, solo a pensare, come fa F., a due dialoghi lucianeschi ben presenti ad Alberti quali la Calumnia o Quomodo historia sit conscribenda. Fu allora l'istinto di verosimiglianza del dialogo d'area cice- 